\title{
Occupation des terres, typologie et structure des faciès de végétation à Vitellaria paradoxa (Gaertn F.) de la Commune de Toukountouna au Nord - Bénin
}

\author{
Vincent Isidore TCHABI ${ }^{*}$ et Koudous Olatundji ADECHI \\ Département de Génie de l'Environnement, Ecole Polytechnique d'Abomey-Calavi, \\ Université d'Abomey-Calavi, 01 BP 2009 Cotonou, Bénin. \\ *Auteur correspondant, E-mail: visidor55@yahoo.fr; Tel: (+229) 97019041 /9595 5553
}

\section{RESUME}

La présente étude, basée sur l'évaluation des changements du couvert végétal au cours des deux dernières décennies (1990 - 2010), a pour la caractérisation structurale et l'évaluation de la dynamique des faciès de végétation à karité, Vitellaria paradoxa (Gaertn F.), de la commune de Toukountouna au Nord-Bénin. Elle est basée sur la cartographie de différents faciès à karité, l'analyse des paramètres structuraux et dendrométriques. Les résultats révèlent d'importantes modifications du couvert végétal et de sa structuration, principalement au détriment des savanes boisées. En vingt ans, les formations naturelles ont perdu $63,13 \%$ de leur superficie avec un taux annuel moyen de 3,15\%. Cette tendance indique que ce sont les champs et jachères à Vitellaria qui seront appelés à caractériser les formes de végétation de l'avenir. La surface terrière la plus élevée s'observe dans les champs et jachères $\left(83,62 \mathrm{~m}^{2} / \mathrm{ha}\right)$ contre $(29,79 \mathrm{~m} / \mathrm{ha})$ dans les formations naturelles. C'est aussi dans les champs et jachères que l'on observe les plus vieux individus (67 tiges/ha) contre (56 tiges/ha) dans les formations naturelles. La conservation durable des faciès est donc dictée par l'homme, à la recherche des terres agricoles.

() 2014 International Formulae Group. All rights reserved.

Mots clés : Vitellaria paradoxa, structure, surface terrière, Toukountouna, Bénin.

\section{INTRODUCTION}

Dans les pays en voie de développement, les populations locales dépendent surtout des Produits Forestiers Non Ligneux pour leur santé primaire, leur besoin nutritionnelle et leur revenu. Au Bénin, les parcs à karité fournissent une part non négligeable de ces produits. Selon les estimations, au moins 150000 tonnes d'amandes sont collectées chaque année (Sanou et Lamien, 2011) ; et presque toutes les parties de cet arbre sont destinées à divers usages: le beurre de karité présente d'importantes propriétés thérapeutiques, en particulier dermatologiques, protège contre les rayons ultraviolets et a des propriétés hydratantes et régénératrices. Au début de la saison des pluies (mai à août), sa pulpe constitue un aliment de base pour les villageois les plus pauvres, qui ont peu de céréales à leur disposition en cette période de soudure (Arbonier, 2005 ; Akoegninou et al., 2006). Sous ces latitudes, le Vitellaria paradoxa est bien adapté à des conditions qui sont généralement peu favorables au palmier à huile, jouant ainsi un rôle de substitut à ce 
dernier (Bioli et Lafleur, 2008). Il est communément associé à des systèmes d'agroforesterie (Saint-Sauveur, 2001 ; Tossou, 2013) ; et bénéficie de l'attention que lui portent les agriculteurs dans les champs. De ce fait, il est protégé par les lois forestières de la plupart des pays compris dans son aire de répartition naturelle. Les parcs à karité doivent donc leur existence et leur structure à l'homme (Diarassouba et al., 2008). Mais il est fréquent de constater l'élimination de l'arbre, notamment lorsque des terres sont défrichées au profit de la culture du coton et de l'igname, de même que pour la production de charbon. Pour dissuader les populations, des études ethnobotaniques de Vitelleria paradoxa ont été entreprises dans toute la zone de distribution de l'espèce, qui ont permis de mettre en évidence leurs rôles culturel, médicinal et pharmacologique. Malgré tous ces avantages, les informations relatives à sa typologie et la structure de ses peuplements, susceptibles de prévoir leur capacité productive et leur statut, ne sont pas disponibles. Dans un tel contexte, l'étude de la connaissance des modifications des faciès de végétation et l'appréciation de leurs paramètres structuraux ne peuvent qu'être recommandées.

\section{MATERIEL ET METHODES}

Milieu d'étude

La Commune de Toukountouna s'étendent sur $1114 \mathrm{~km}^{2}$, soit $1,42 \%$ de la superficie du pays, compte trois arrondissements: Toukountouna centre (318 $\mathrm{km}^{2}$ ), Tampégré $\left(203 \mathrm{~km}^{2}\right)$ et Kouarfa (593 $\mathrm{km}^{2}$ ) et regroupe 22 villages. Elle est délimitée au nord-ouest par la commune de Tanguiéta, au nord-est par la commune de Kouandé, au sud par les communes de Natitingou et de Kouandé (Figure 1).

Le climat y est du type soudanien avec une saison de pluies de cinq (05) mois (mimai à mi-octobre) et une saison sèche de sept
(07) mois (mi - octobre à mi - mai). Les précipitations varient entre 1000 et $1200 \mathrm{~mm}$. Les mois d'août et de septembre sont les plus pluvieux et les mois d'avril et mai, les plus chauds. On y note des signes de perturbation climatique caractérisés entre autres par le démarrage tardif des pluies, des poches de sécheresse en cours de saison pluvieuse, se traduisant par des arrêts brusques de précipitations. Ce phénomène est général dans le département de l'Atacora dont fait partie cette commune. La température moyenne est d'environ $27{ }^{\circ} \mathrm{C}$ avec des variations de $17{ }^{\circ} \mathrm{C}$ à $38{ }^{\circ} \mathrm{C}$. Les amplitudes thermiques sont fortes entre le jour et la nuit surtout pendant la période de l'harmattan. Ce climat est favorable au développement du karité Vitellaria paradoxa.

Le relief y est caractérisé par une succession de plateaux et de plaines à l'Est, de montagnes à l'Ouest, et de vallées au Centre. Des pentes relativement fortes, surtout du côté ouest, posent des problèmes d'érosion des sols et de dégradation des pistes de desserte rurale. Les altitudes varient entre 380 et 590 m. Un tel relief est très propice à l'occupation humaine, suscitée par les faveurs du climat de la région.

Les sols sont principalement du type ferrugineux tropical, ce qui fait qu'il est noté une forte dominance de la savane arborée et arbustive. Les espèces qui dominent les terroirs sont le baobab (Adansoniadigitata), le karité (Vitellaria paradoxa), le néré (Parkiabiglobosa), le caïlcédrat (Khayasenegalensis), le tamarinier (Tamarindusindica), le faux karité (Lophira lanceolata), le fromager (Bligiasapinda).

\section{Matériel végétal}

Le Vitellaria paradoxa est une espèce autochtone d'Afrique de l'Ouest (Akoegninou et al., 2006). Il est présent du Sénégal jusqu'en Ouganda, à des latitudes comprises entre $9^{\circ}$ et $14^{\circ} \mathrm{N}$ en Afrique de $1^{\prime}$ Ouest, $7^{\circ}$ et $12^{\circ} \mathrm{N}$ en Afrique centrale, $2^{\circ}$ et $8^{\circ} \mathrm{N}$ en 
Afrique de l'Est. Cette zone correspond aux parties centrale et méridionale plus humides des pays secs (Burkina Faso, Mali et Niger) et à la partie septentrionale plus sèche des pays humides (Bénin, Cameroun, Côte d'Ivoire, Ghana, Nigeria Togo et Ouganda).

Le Vitellaria paradoxa est présent dans les savanes humides et sèches où les précipitations annuelles oscillent entre 400 et $1500 \mathrm{~mm}$. Il est adapté à une grande variété de conditions environnementales. Il constitue des peuplements dominants dans les savanes soudaniennes, en association avec d'autres espèces comme le faux karité (Lophira lanceolata), le badamier (Terminalia macroptera et Terminalia avicennioides) et le néré (Parkia biglobosa). Il est communément associé à des systèmes d'agroforesterie et bénéficie de l'attention que lui portent les agriculteurs dans les champs. L'arbre atteint une hauteur de 10 à $20 \mathrm{~m}$. Il a une forme de parasol, de pyramide ou de globe. Le tronc cylindrique des arbres adultes mesure entre 30 et $80 \mathrm{~cm}$ de diamètre. Le fruit est une baie composée d'une pulpe nutritive qui entoure une noix relativement grosse. Celle-ci contient une amande riche en huile, d'où le beurre de karité est extrait. Le genre Vitellaria regroupe deux espèces à savoir nilotica, originaire d'Afrique de l'Est, et paradoxa, originaire d'Afrique de l'Ouest, qui diffèrent par des caractères morphologiques tels que la taille de la fleur. Ce sont des espèces essentiellement zoochores qui se multiplient par pollinisation croisée.Les principaux pollinisateurs sont les abeilles, les mouches, les fourmis et les guêpes. Les graines sont dispersées principalement par les humains, les singes et les oiseaux.

\section{Collecte et traitement des données Cartographie des différents faciès de végétation}

Elle a consisté d'abord au traitement des images satellitaires. Le prétraitement et ensuite le traitement constituant un travail préliminaire qui se fait avant l'interprétation proprement dite. A ce stade, il a été question de procéder à trois principales opérations à l'aide du logiciel Erdas Imagine 8.7. Il s'agit de la correction géométrique ou rectification, de l'amélioration des couleurs et de la composition colorée. Elle a permis de cartographier toutes les unités d'occupation du sol et d'évaluer leur superficie. Le traitement des données relatives aux caractéristiques structurales des peuplements a été fait à l'aide des logiciels minitab 14 et Canoca 4.5. La collecte de données a été faite sur la base de la Carte d'Occupation des Sols de la commune, qui a servi de fond pour cibler les différents faciès de végétation. Des relevés ont par la suite été réalisés sur des placeaux rectangulaires de $1000 \mathrm{~m}^{2}$ pour tenir compte des travaux de Houehanou et al., 2012. Viennent ensuite les mesures dendrométriques, effectuées sur 31 placeaux, dont 26 sont situés dans les champs et jachères $(\mathrm{CJ})$ et 5 dans les formations végétales naturelles, c'est-à-dire les forêts claires et savanes (F\&SA) du fait de la faible surface occupée par les formations naturelles, systématiquement détruites à des fins agricoles.

Ces mesures dendrométriques sont effectuées sur tous les arbres dont le dbh (diameter at breast height) $\geq 10 \mathrm{~cm}$ à $1,30 \mathrm{~m}$ au-dessus du sol.

Les données recueillies (diamètre à 1,30 $\mathrm{m} \mathrm{du}$ sol et hauteur totale) ont permis de calculer :

- la densité, évaluée en nombre d'individus à l'hectare et qui concerne les arbres dont le dbh est supérieur ou égal à 10 $\mathrm{cm}$

$$
\text { - le diamètre moyen }(\mathrm{dm})=\frac{1}{\mathrm{n}} \sum d_{\mathrm{i}}
$$

L'étape suivante a consisté à calculer la surface terrière $(G)$ : c'est la somme des sections des arbres de $\mathrm{dbh} \geq 10 \mathrm{~cm}$ mesurés sur écorce à $1,30 \mathrm{~m}$ du sol. Elle s'exprime en $\mathrm{m}^{2} /$ ha et correspond, pour chaque groupement végétal décrit, à la moyenne des surfaces 
terrières des plateaux les plus représentatifs. Elle se calcule à partir de la formule : $\mathbf{G}=$ $\Sigma$ gi, avec gi $=\frac{\pi}{4} \mathbf{x} \mathbf{d}^{2}$, dans laquelle $\mathrm{d}_{\mathrm{i}}$ est le diamètre de l'arbre i. La surface terrière est en effet un bon outil de classification de maturité des populations végétales. Pour Sokponet al., 2006, le diamètre de l'arbre moyen est un paramètre d'intérêt très pratique qui est souvent recommandé en sylviculture; d'où l'importance accordée à ce paramètre. L'ensemble des arbres mesurés dans chaque peuplement est par la suite réparti dans des classes de diamètre et représenté par des histogrammes, soit sur la base des surfaces terrières, soit sur la base des fréquences. La répartition par classes de diamètre sur la base des fréquences d'observation des individus a été ajustée à la distribution de Weibull à trois paramètres en raison de sa flexibilité (Bonou et al., 2009) où la fonction de densité (F) a été exprimée en fonction du diamètre $(\mathrm{x})$ suivant

la formule $\mathbf{F}(\mathbf{x})=\frac{c}{b}\left(\frac{x-a}{b}\right)^{c-1} e^{-\left[\frac{x-2}{b}\right]^{c}}$, dans laquelle :

$\mathrm{a}=$ paramètre de position avec $a=10 \mathrm{~cm}$ pour les diamètres; $b=$ paramètre d'échelle ou de taille et $c=$ paramètre de forme liée à la structure observée.

La répartition par classes de diamètre sur la base des surfaces terrières est ajustée à des fonctions mathématiques appropriées qui établissent la relation entre la hauteur totale et le diamètre à hauteur d'homme de tous les arbres et arbuste mesurés. C'est la relation allométrique de croissance. La croissance allométrique implique l'existence d'une relation curvilinéaire entre les deux paramètres mesurés et constitue un bon indicateur des conditions écologiques de croissance de l'espèce. Elle s'exprime par l'équation $\mathrm{Y}=\mathrm{a} \cdot \operatorname{Ln}(\mathrm{X})+$ bavec $\mathrm{Y}=$ hauteur de l'arbre et $\mathrm{X}=\mathrm{dbh}$; $\mathrm{a}$ et $\mathrm{b}$ sont des constantes et b est appelé le coefficient d'allométrie. Elle est matérialisée par une représentation graphique des hauteurs et des diamètres.

La structure spatiale est également caractérisée. Elle correspond à un type de répartition au sol de tous les individus d'une communauté. Elle se fonde sur la variance et la moyenne des échantillons (quadrats ou placeaux) étudiés. Trois types de distribution sont utilisées pour caractériser les communautés végétales: la distribution aléatoire ou poissonnienne dans laquelle la variance est égale à la moyenne; la distribution régulière avec une variance inférieure ou égale à la moyenne) et la distribution agrégative ou contagieuse quand la variance est supérieure à la moyenne. L'indice de dispersion utilisé pour caractériser la distribution spatiale des individus est celui de Blackman (IB) à cause de sa facilité d'emploi. Il a pour formule : $\mathbf{I B}=\boldsymbol{\sigma}^{2} / \mathbf{m}$ avec $\sigma^{2}=$ variance et $m=$ moyenne. IB = 1 quand la distribution est poissonnienne, $<1$ lorsque la distribution régulière et > 1 pour distribution est agrégative.

$\mathrm{La}$ relation allométrique entre le diamètre (D) et la hauteur $(\mathrm{H})$ des arbres dans les formations végétales est traduite dans tous les faciès de végétation par une relation linéaire en coordonnées simples et rapportée à une ligne de référence $\mathrm{H}=100 \mathrm{D}$, qui est une ligne de référence et non une courbe de régression. Dans le cas des arbres du présent, on a $\mathrm{H}<100 \mathrm{D}$ et $\mathrm{H}>100 \mathrm{D}$ dans le cas des arbres du futur. La ligne de référence $\mathrm{H}=$ 100D nous renseigne également sur la physionomie de la formation étudiée. 


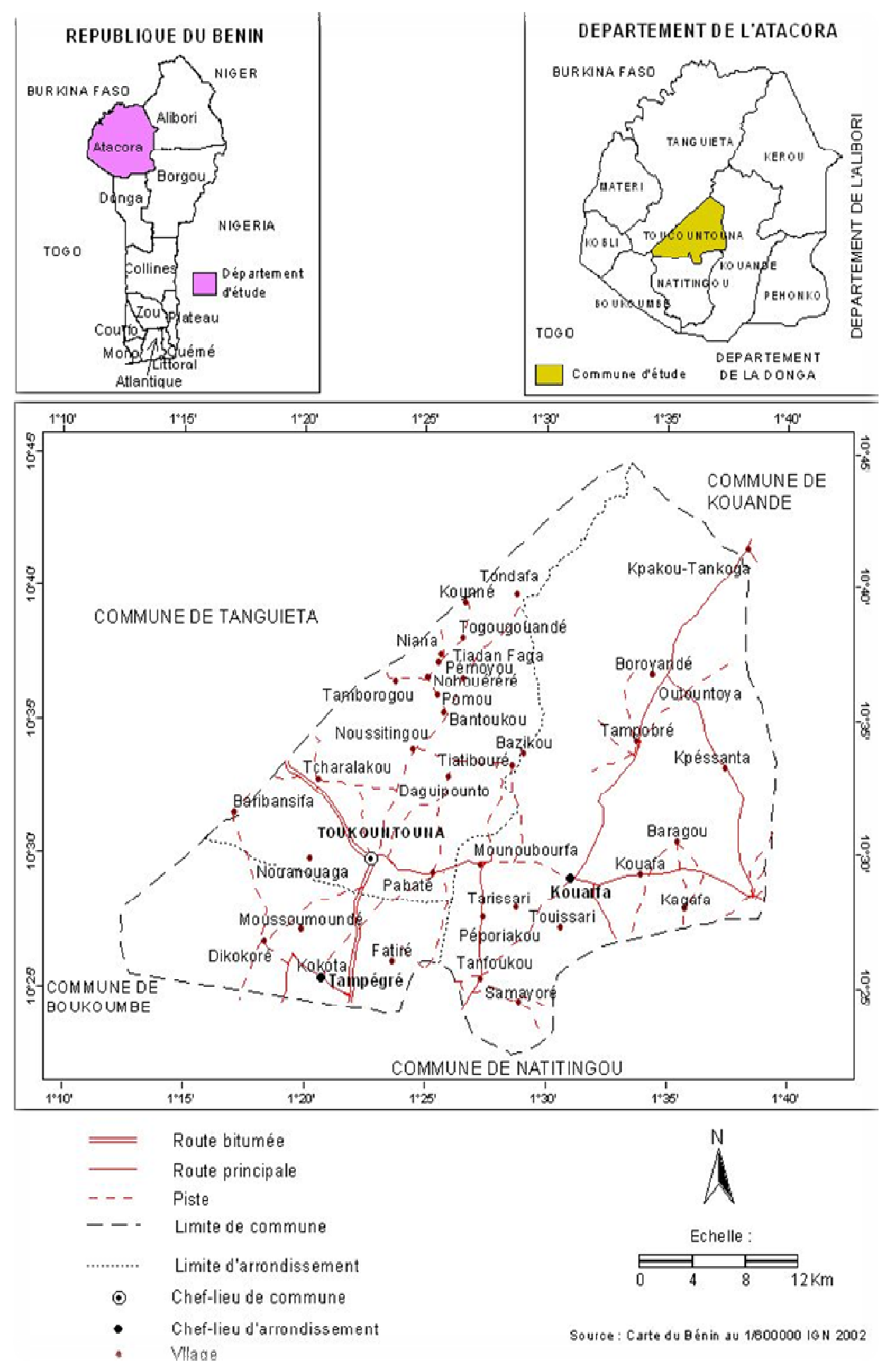

Figure 1: Carte du milieu d'étude. 


\section{RESULTATS}

Dynamique d'occupation des sols de 1990 à 2010

Comparaison des différents paramètres structuraux des deux types de peuplement

Le Tableau 1 présente une synopse des différents paramètres étudiés, qui indique qu'en dehors de l'indice de Blackman, tous les autres paramètres sont plus prépondérants dans les champs et jachères que dans les savanes et forêts claires.

\section{Occupation en 1990}

Les différents types d'occupation de la commune de Toukountouna couvrent une superficie de 107500 ha en 1990 (Tableau 2). L'étude de l'occupation du sol révèle l'existence de différentes formations et formes de végétation en 1990. La lecture du Tableau 2 indique une forte proportion de savanes arborées et arbustives (68\%) suivies des forêts claires et savanes boisées (19\%), de champs et jachères (9\%), des forêts galeries $(2,20 \%)$, puis des agglomérations $(0,68 \%)$.

\section{Occupation en 2010}

Le Tableau 3 présente les superficies occupées par formation végétale en 2010. Les données qui attirent l'attention concernent les cultures et jachères (avec 65\% d'occupation), les savanes arborées et arbustives (18\% d'occupation), les forêts claires et savanes boisées (13\%).

Le Tableau 4 présente les changements intervenus dans l'occupation du sol de 1990 à 2010 et indique que la plus forte régression de couverture végétale concerne les savanes arborées et arbustives (-54579 ha), suivies des forêts galeries (-104ha). Ces données expliquent la forte progression des zones artificialisées: il y a eu certes un effet de régénération avec 154 ha de plantation, mais les cultures et jachères ont occupé 59680 ha et les agglomérations, 1207 ha (Tableau 4).

\section{Structure spatiale des peuplements}

Deux types de distribution se distinguent nettement et rendent compte du degré d'artificialisation ou de dégradation des peuplements. Dans les champs et jachères, la valeur de l'Indice de Blackman est de 2,82 bits, traduisant une distribution agrégative. Dans les formations végétales naturelles, il est de 0,05 bits, qui indique une distribution du type régulier des individus.

Densité des différents peuplements et répartition des individus par classe de diamètres

La Figure 2 présente la répartition par classe de diamètre des individus de Vitellaria paradoxa pour $\mathrm{dbh} \geq 10 \mathrm{~cm}$ dans les champs et jachères puis dans les formations végétales naturelles (savanes et forêts claires).

La densité des individus de cette espèce est de 67 tiges/ha dans les champs et jachères et de 56 tiges/ha dans les savanes et les forêts claires. Dans les champs et jachères, l'équation issue de cette répartition s'exprime par la fonction $\mathrm{f}_{\mathbf{C J}}(\mathbf{x})$ $=0,021\left(\frac{x-10}{70,20}\right)^{0,51} \exp -\left[\frac{x-10}{70,20}\right]^{1,51}$; alors que dans les formations naturelles (forêts claires et savanes), la répartition découle de la fonction :

$f_{\mathrm{F} \& S A}(x)=0,038\left(\frac{x-10}{50,71}\right)^{0,94} \exp -\left[\frac{x-10}{50,71}\right]^{1,94}$

Cependant, on peut observer que la structure diamétrale des individus dans les champs et jachères d'une part et celle des formations naturelles d'autre part se présente sous la forme de «cloche» (Figure 2) et s'ajuste globalement à la distribution de Weibull avec la valeur du paramètre de forme respectivement égale à 1,51 bits et 1,93 bits. Cette distribution est centrée sur les individus appartenant à la classe de diamètre $15 \mathrm{~cm}$, qui traduit 
la prédominance d'individus jeunes ou de petits diamètres dans les deux types de peuplement.

\section{Surface terrière et diamètre de l'arbre de surface terrière moyenne}

Les Figures 3 et 4 montrent la marche de la surface terrière des individus des deux types de peuplements en fonction des classes diamétrales. La surface terrière des populations des champs et jachères s'ajuste par une fonction polynominale d'équation: $y=-0,1056 x^{3}+1,3474 x^{2}-4,1672 x+3,4541$ Celle des peuplements des formations naturelles (forêts claires et savanes) s'ajuste aussi par une fonction polynomiale d'équation: $\mathrm{y}=-0,0017 \mathrm{x}^{3}+0,0166 \mathrm{x}^{2}+$ $0,335 x-0,4003$.

$\mathrm{Au}$ niveau des individus des peuplements des champs et jachères, la surface terrière varie d'une placette à une autre de $0,05 \mathrm{~m}^{2} /$ ha à $4,30 \mathrm{~m}^{2} /$ ha et la moyenne obtenue est de $36,82 \mathrm{~m}^{2} / \mathrm{ha}$. La surface terrière de l'arbre moyen est égale à $2,20 \mathrm{~m}^{2} /$ ha et le diamètre de l'arbre de surface terrière moyenne est de 83,62 cm. Dans ces peuplements de savanes et forêts claires, la surface terrière varie d'une placette à une autre de $0,05 \mathrm{~m}^{2} /$ ha à $4,68 \mathrm{~m}^{2} /$ ha et la moyenne obtenue est de $15,62 \mathrm{~m}^{2} / \mathrm{ha}$. La surface terrière de l'arbre moyen est égale à $0,27 \mathrm{~m}^{2} /$ ha et le diamètre de l'arbre de surface terrière moyenne est de $29,79 \mathrm{~cm}$.

On note un accroissement de la surface terrière quand on part des forêts claires et savanes $\left(15,62 \mathrm{~m}^{2} / \mathrm{ha}\right)$ vers les champs et jachères $\left(36,82 \mathrm{~m}^{2} / \mathrm{ha}\right)$. Le diamètre de l'arbre de surface terrière moyenne suit la même tendance et est en effet plus élevé dans les champs et jachères que dans les formations naturelles soit: $83,62 \mathrm{~cm}$ contre $29,79 \mathrm{~cm}$ (Tableau 1).

Le pic de la surface terrière se retrouve à $135 \mathrm{~cm}$ dans les champs et jachères et $75 \mathrm{~cm}$ dans les formations naturelles.

Selon Natta et al. (2011), le pic de la répartition de la surface terrière par classe de diamètres est un critère de choix pour apprécier le stade d'évolution et des conditions d'exploitation des peuplements forestiers. En général, le pic de la courbe de répartition des surfaces terrières croît lorsqu'on passe des forêts secondaires jeunes aux forêts secondaires vieilles puis aux forêts primaires. Les individus des champs et jachères sont donc plus vieux que ceux des forêts claires et savanes.

En prenant donc comme indicateur l'évolution de la surface terrière, on retiendra que c'est dans les champs et jachères que la production de noix est actuellement la plus forte, parce que plus constitués de vieux individus qui présentent les productivités les plus élevées. Mais la production dans le futur sera déterminée par les individus des peuplements naturels (forêts claires et savanes).

\section{Relation Hauteur - Diamètre (H/D)}

La relation allométrique de croissance existant entre les arbres recensés dans l'ensemble des peuplements est illustrée par les diagrammes hauteur-diamètre (H/D) des Figures 5 et 6 . Cette relation se présente sous l'équation $\mathrm{H}=$ 2,6007+0,0697D dans les champs et jachères et $\mathrm{H}=1,7447+0,0817 \mathrm{D}$ dans les forêts claires et savanes.

De 1'analyse des Figures 5 et 6, on retient que $\mathrm{H}=100 \mathrm{D}$ est une ligne de référence, et non une courbe de regression. Ainsi, que ce soit dans les champs et jachères ou dans les formations naturelles, on a des arbres du présent, ce qui augure d'une production soutenue de noix de karité dans les deux cas de figure. 
Tableau 1: Résumé comparatif des paramètres structuraux des différents faciès à Vitellaria paradoxa.

\begin{tabular}{lcc}
\hline $\begin{array}{l}\text { Paramètres calculés/Type de } \\
\text { formation }\end{array}$ & $\begin{array}{c}\text { Champs et } \\
\text { jachères } \\
\text { (CJ) }\end{array}$ & $\begin{array}{c}\text { Forêts claires et savanes } \\
\text { (F\&SA) }\end{array}$ \\
\hline Densité (Nombre de tiges/ha) & 67 & 56 \\
surface terrière (m²/ha) & 36,82 & 15,62 \\
Diamètre de l'arbre moyen (en cm) & 83,62 & 29,79 \\
Indice de Blackman (en bits) & 2,82 & 0,05 \\
Paramètre de forme (en bits) & 1,51 & 1,93 \\
\hline
\end{tabular}

Tableau 2 : Superficie des unités d'occupation des sols en 1990.

\begin{tabular}{lccc}
\hline Formation & Code & Superficie en 1990 (ha) & Proportion (1990) \% \\
\hline Forêt galerie & FG & 2370 & 2,20 \\
Forêt claire et savane boisée & FCSB & 20813 & 19 \\
Savane arborée et arbustive & SAA & 73507 & 68 \\
Plantation & PLT & 102 & 0,09 \\
Cultures et jachères & CJ & 9838 & 9 \\
Agglomération & AGG & 727 & 0,68 \\
Plan d'eau & PE & 7 & 0 \\
Total & & $\mathbf{1 0 7 5 0 0}$ & $\mathbf{1 0 0}$ \\
\hline
\end{tabular}

Tableau 3: Superficie des unités d'occupation des sols en 2010.

\begin{tabular}{lccc}
\hline Formation & Code & $\begin{array}{c}\text { Superficie en 2010 } \\
\text { (ha) }\end{array}$ & Proportion (2010) \% \\
\hline Forêt galerie & FG & 2266 & 2,11 \\
Forêt claire et savane boisée & FCSB & 14455 & 13 \\
Savane arborée et arbustive & SAA & 18928 & 18 \\
Plantation & PLT & 256 & 0,24 \\
Cultures et jachères & CJ & 69518 & 65 \\
Agglomération & AGG & 1934 & 2 \\
Plan d'eau & PE & 7 & 0 \\
Total & & $\mathbf{1 0 7 5 0 0}$ & $\mathbf{1 0 0}$ \\
\hline
\end{tabular}


Tableau 4: Evolution des unités d'occupation des sols au sein de la même classe (période: 1990 2010).

\begin{tabular}{|c|c|c|c|c|}
\hline Formation & Code & $\begin{array}{c}\text { Superficie en } \\
1990 \text { (ha) }\end{array}$ & $\begin{array}{l}\text { Superficie en } \\
2010 \text { (ha) }\end{array}$ & $\begin{array}{c}\text { Changement } \\
(1990-2010) \\
\text { (ha) } \\
\end{array}$ \\
\hline Forêt galerie & FG & 2370 & 2266 & -104 \\
\hline $\begin{array}{l}\text { Forêt claire et savane } \\
\text { boisée }\end{array}$ & FCSB & 20813 & 14455 & -6358 \\
\hline $\begin{array}{l}\text { Savane arborée et } \\
\text { arbustive }\end{array}$ & SAA & 73507 & 18928 & -54579 \\
\hline Affleurement rocheux & AFFL & 136 & 136 & 0 \\
\hline Plantation & PLT & 102 & 256 & 154 \\
\hline Cultures et jachères & CJ & 9838 & 69518 & 59680 \\
\hline Agglomération & AGG & 727 & 1934 & 1207 \\
\hline Plan d'eau & $\mathrm{PE}$ & 7 & 7 & 0 \\
\hline Total & & 107500 & 107500 & \\
\hline
\end{tabular}

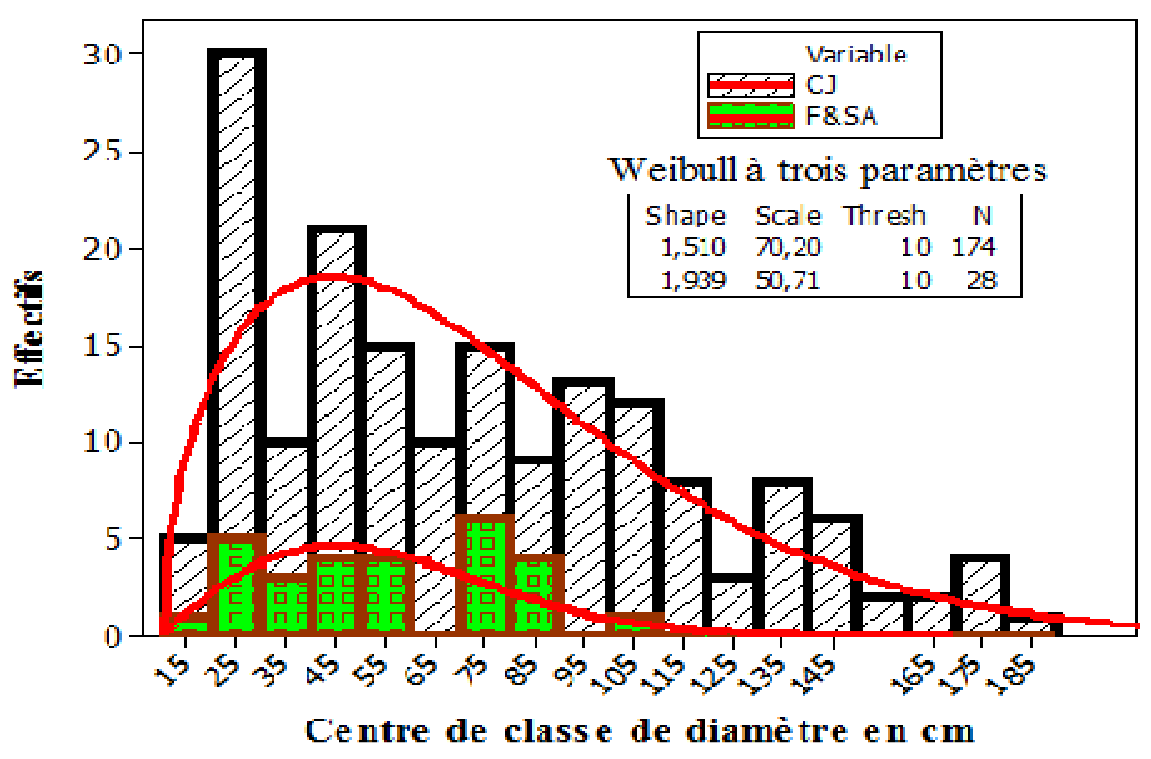

Figure 2 : Répartition par classes de diamètre des individus dans les différents peuplements. $\mathrm{CJ}=$ champs et jachères ; F\&SA = forêts claires et savanes. 


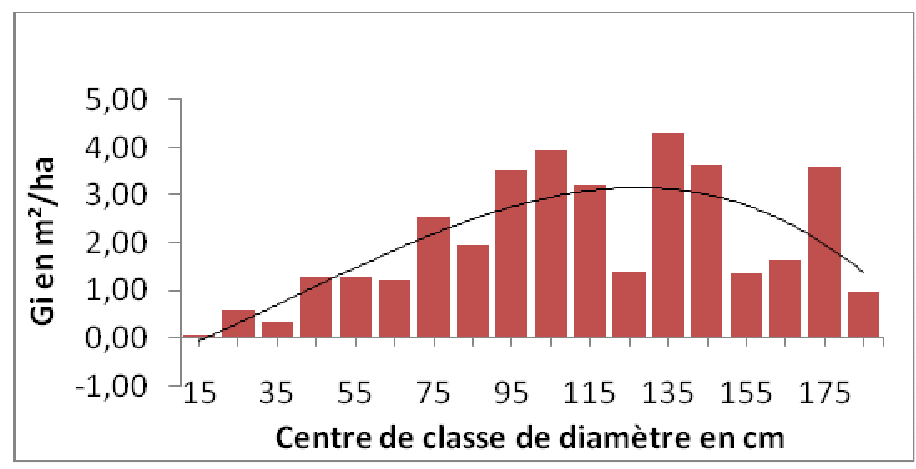

Figure 3 : Marche de la surface terrière des individus dans les champs et jachères en fonction des classes de diamètre.

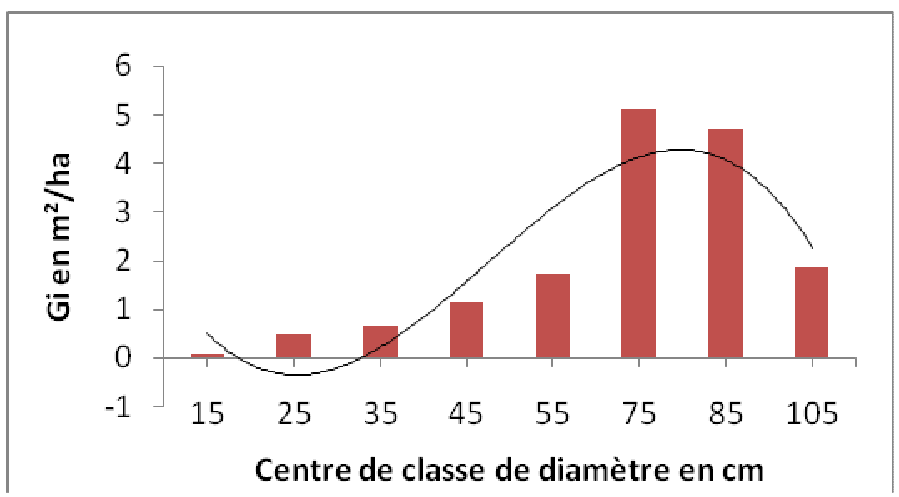

Figure 4 : Marche de la surface terrière des individus dans les formations naturelles en fonction des classes de diamètre.

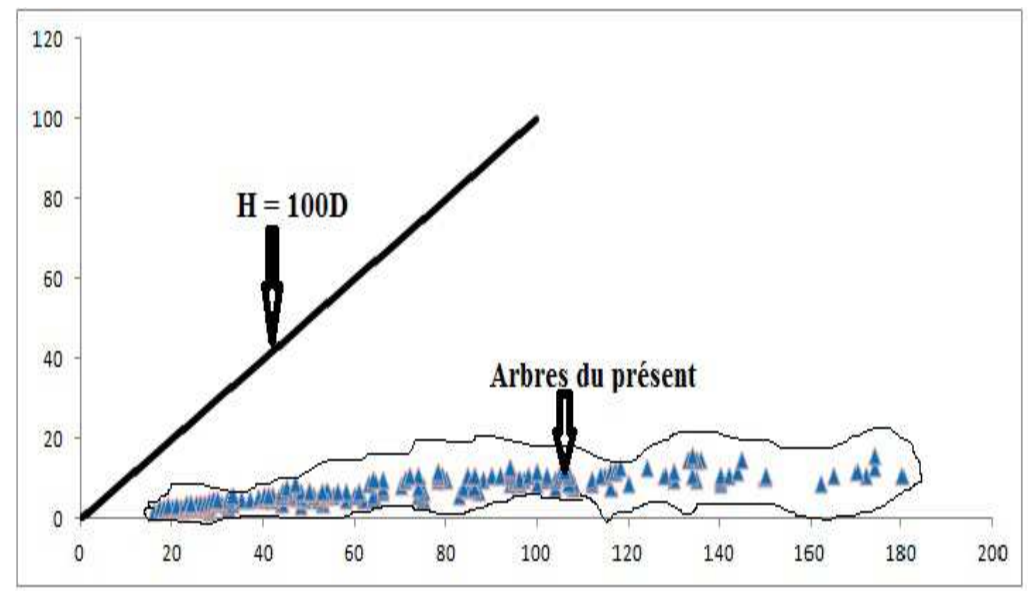

Figure 5 : Relation hauteur-diamètre des individus dans les champs et jachères. 


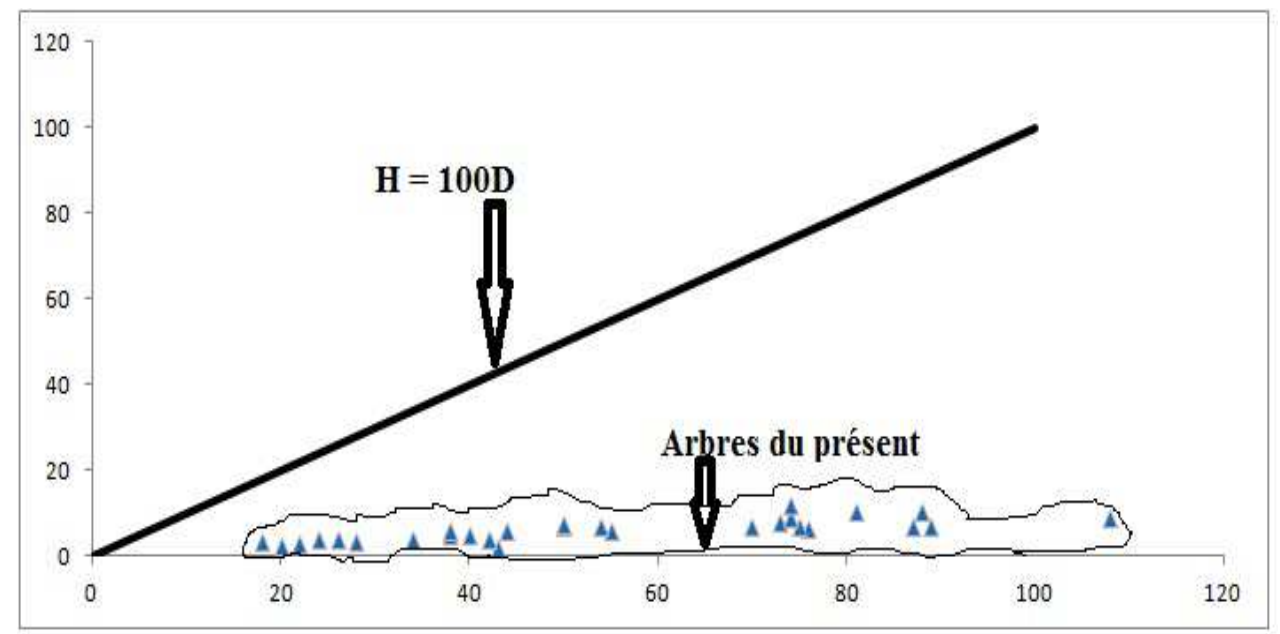

Figure 6 : Relation hauteur - diamètre des individus dans les formations végétales naturelles.

\section{DISCUSSION}

\section{Au sujet de la structure des peuplements}

Les peuplements de Vitellaria de la commune de Toukountouna ne présentent pas la même structure. Ce qui reste maintenant à clarifier, c'est la cause de cette variation structurale, qui, et c'est important, influence la production de noix de karité. Pour Boffa (2000), la répartition des ligneux est le plus fréquemment groupée qu'aléatoire. Ils soulignent que ce mode de répartition s'explique par plusieurs raisons à savoir : l'hétérogénéité du substrat, le tempérament des espèces et le mode de dissémination des graines. Natta et al. (2011) soulignent aussi que la distribution agrégative semble liée au mode de dissémination et au tempérament des espèces. Il explique ce mode de répartition spatiale par la prédominance des zoochores et plus précisément par le fait que les agents disséminateurs (animaux) déposent souvent les semences à côté des arbres fruitiers, de leurs terriers, ou de leurs niches; ce qui engendre une forte concentration de semences et par conséquent une distribution agrégative. Sans remettre en cause ces explications, on retiendra dans le cadre de cette étude que, toute chose égale par ailleurs, c'est le degré d'artificialisation (ou de mise en culture des peuplements) qui explique la structure agrégée. L'apport de cette étude sera donc d'intégrer l'indicateur "forme de végétation" dans le type structural des peuplements de Vitellaria; la forme de végétation étant la combinaison de la formation végétale et de son degré d'artificialisation. Il s'agit d'agroécosystèmes (Agbahungba, 2007) dans lesquels l'agriculture, l'élevage et la foresterie resteront des activités clefs (quoique mal rétribuées).

\section{Au sujet de la dynamique spatio-temporelle des unités d'occupation du sol}

Les peuplements naturels de la commune ont été excessivement détruits et ont laissé place surtout aux espaces agricoles. Dans l'ensemble, entre 1990 et 2010 les formations naturelles ont trouvé leur superficie réduite de plus de $109 \%$, soit 61041 hectares. La démographie sans cesse galopante de la commune de Toukountouna, les pratiques agricoles du type extensif seraient surtout à la base de cette régression des espaces forestiers. Cette observation corrobore celle de Mama et Oloukoï (2003), Oloukoï et al. (2006), qui s'explique par une pratique culturale, obsolète, destructrice de la biodiversité. Ces résultats corroborent aussi ceux de Gomina (2011), Detondé (2012) et Lawin (2012) qui estiment un taux de destruction assez élevé pouvant permettre la destruction totale des formations naturelles. 
Le seul prétexte au vu des besoins des populations, pour conserver les formations naturelles, reste la préservation de la biodiversité (MEHU/PNUD, 2002 ; Djégo, 2004).

\section{Conclusion}

$\mathrm{Au}$ terme de la présente étude, l'approche synchronique a révélé une faible densité au niveau des deux populations de $V$. paradoxa étudiées. Mais le véritable problème, révélé par l'approche diachronique, est que l'espace agricole y a largement pris le dessus sur les formations végétales naturelles avec un risque de disparition de toutes les formations spontanées. Les populations des champs et jachères sont vieillissantes et ne peuvent assurer la durabilité de la production des amandes de karité, la régénération naturelle dans les champs et jachères étant compromise du fait des activités annuelles de nettoyage des champs et les feux de végétation.

\section{REFERENCES}

Agbahungba AG. 2007. Dynamique de la matière organique et influence de la fertilisation dans les systèmes agroforestiers du Sud-Bénin. Thèse de doctorat, Univ. de Cocody-Abidjan, Côte d'Ivoire, 233p.

Akoegninou A, Van der Burg WJ, Van der Maesen LJG. 2006. Flore Analytique du Bénin. Université d'Abomey Calavi : Cotonou, Bénin ; 1034 p.

Arbonier M. 2000. Arbres Arbustes et Lianes des Zones Sèches d'Afrique de l'Ouest. CIRAD.

Bioli M, Lafleur M. 2008. Renforcement des Capacités des Productrices de Beurre de Karité en Afrique de l'Ouest. CECI : Montréal ; 110.

Boffa JM. 2000. Les parcs agroforestiers en Afrique de l'Ouest: clés de la conservation et d'une gestion durable.Unasylva, 51: 11 - 12.

Bonou W, GlèlèKakaï R, Assogbadjo AE, Fonton HN, Sinsin B. 2009. Characterisation of Afzelia africana Sm.
Habitat in Lama forest reserve of Benin. Forest Ecology and Management, 676: 19.

Detondé J. 2012. Analyse cartographique de la dynamique spatiotemporelle de la forêt classée de TTK après l'aménagement. Mémo. DESS, UP., 55 p.

Diarassouba N, Koffi KE, N'Guessan KA, Van Damme P, Sangare A. 2008. Connaissances locales et leur utilisation dans la gestion des parcs à karité en Côte d'Ivoire. Afrika Focus, 21: 77 - 96.

Gomina SI. 2011. Structure et Dynamique des Forêts Classées de Djougou. Mémoire de Master, FSA/UAC 157 p.

Houehanou TD, GlèlèKakaï RL, Assogbadjo AE, Kindomihou V, Houinato M, Witting R, Sinsin BA. 2012. Change in the woody floristic composition, diversity and structure from protected and unprotected savannahs in Pendjari Biosphere Reserve (Benin, West Africa). AJE, 12046B: 1 7.

Lawin D. 2012. Dynamique spatio-temporelle des Bouquets de la commune de Ouaké. Mémo. DESS, UP, 150 p.

Mama VJ, Oloukoi J. 2003. Evaluation de la précision des traitements analogiques des images satellitaires dans l'étude de la dynamique de l'occupation du sol. Télédétection, 3(5): 429 - 441.

MEHU/PNUD. 2002. Stratégies nationales et plan d'actions pour la conservation de la diversité biologique. Bénin, 197p.

Natta AK. Adomou AC, Tchabi VI, Sogbegnon GA, Mensah GA, Sinsin BA. 2011. Inventaire, typologie et structure des populations naturelles de Pentadesma butyracea (Clusiaceae) de la chaîne d'Ataccora au Nord-Ouest du Bénin. Bulletin de Recherche Agronomique $d u$ Bénin, 70 : 10 - 24.

Oloukoï J, Mama VJ, Agbo FB. 2007. Modélisation de la dynamique de l'occupation des terres dans le Département des collines au Bénin. Télédétection, 6(4): 305 - 323

Saint-Sauveur A. 2001. The indigenous management techniques of farmed 
parklands. » In Teklehaimanot, Z., 2001.

Third annual report of improved management of agroforestry parkland systems in Sub-Saharan Africa, pp 84 92.

Sanou H, Lamien N. 2011. Vitellaria paradoxa, Karité. Conservation et Utilisation Durable des Ressources Génétiques des Espèces Ligneuses Alimentaires Prioritaires de l'Afrique Subsaharienne. Bioversity International: Rome Saforgen; 12.
Sokpon N, Biaou SH, Ouinsavi C, Hunhyet O. 2006. Bases techniques pour une gestion durable des forêts claires du Nord-Bénin : rotation, diamètre minimal d'exploitabilité et régénération. Bois et Forêts des Tropiques, 287(1): 45 - 57.

Tossou MV. 2013. Contribution des jachères à la conservation de Pterocarpus erinaceus (Poir) et d'Afzelia africana (smith ex pers.) dans la commune de DasssaZounmè au Centre-Bénin. Mémoire DEA, Univ. d'Abomey-Calavi, Bénin, $45 \mathrm{p}$. 